\title{
Optimal Investment-Reinsurance Strategies for Insurers with Mean-Reversion and Mispricing under Variance Premium Principle
}

\author{
Yuzhen Wen \\ School of Mathematical Sciences, Qufu Normal University, Qufu, China \\ Email: wenyzhen@163.com
}

How to cite this paper: Wen, Y.Z. (2018) Optimal Investment-Reinsurance Strategies for Insurers with Mean-Reversion and Mispricing under Variance Premium Principle. Applied Mathematics, 9, 806-820. https://doi.org/10.4236/am.2018.97056

Received: June 25, 2018

Accepted: July 22, 2018

Published: July 25, 2018

Copyright $\odot 2018$ by author and Scientific Research Publishing Inc. This work is licensed under the Creative Commons Attribution International License (CC BY 4.0).

http://creativecommons.org/licenses/by/4.0/

\section{(c) (i) Open Access}

\begin{abstract}
This paper considers a robust optimal reinsurance-investment problem for an insurer with mispricing and model ambiguity. The surplus process is described by a classical Cramér-Lunderg model and the financial market contains a market index, a risk-free asset and a pair of mispriced stocks, where the expected return rate of the stocks and the mispricing follow mean reverting processes which take into account liquidity constraints. In particular, both the insurance and reinsurance premium are assumed to be calculated via the variance premium principle. By employing the dynamic programming approach, we derive the explicit optimal robust reinsurance-investment strategy and the optimal value function.
\end{abstract}

\section{Keywords}

Proportional Reinsurance, Robust Control, Optimal Investment Strategy, Utility Function, Mispricing

\section{Introduction}

Reinsurance and investment are the main tools for insurers to manage the risk and profit. Insurers can transfer the risk to reinsurers by purchasing reinsurance; meanwhile, they would invest their surplus into financial market to pursue extra profit. Recently, the problem of optimal reinsurance and investment has attracted great interest. For example, Schmidli [1], Bai and Guo [2] and Chen et al. [3] investigated the optimal investment-reinsurance strategies for insurers to minimize the ruin probability; Bäuerle [4], Bai and Zhang [5], Zeng and Li [6] studied the optimal reinsurance and investment strategies for insurers with mean-variance criteria; Maximizing the expected utility from terminal wealth 
was investigated by many literature, see among Yang and Zhang [7], Wang [8], $\mathrm{Xu}$ et al. [9] and so on.

On the other hand, it is a common belief that there is no agreement on which model, or real world probability, should be used in practice. So model uncertainty exists widely in the field of portfolio selection. Thus, some scholars have advocated and investigated the effect of model uncertainty on portfolio selection. Robust decision making in the portfolio context is introduced by Maenhouut [10]. A number of other papers are built on Maenhouut [10] to address the implications of ambiguity on portfolio choice. Liu et al. [11] discussed the robust consumption and portfolio choice for time-varying investment opportunities. Yi et al. [12] focus on an optimal portfolio selection problem under SV model with model uncertainty. For more details, we refer the reader to Li et al. [13], Sun et al. [14] and references therein.

Theoretically, real markets are full of friction. Hence, there exists mispricing between a pair of assets. We can find vivid examples of mispricing in certain Chinese companies (such as Bank of China) traded on both Chinese stock exchanges as share A and Hong Kong stock exchanges as shares. Yi et al. [15] first consider a dynamic portfolio problem with mispricing and model ambiguity. $\mathrm{Gu}$ et al. [16] discuss optimal proportional reinsurance-investment problem for an insurer with mispricing and model ambiguity. In this paper, we seek the optimal proportional reinsurance-investment problem with mispricing under observed mean-reverting stochastic risk premium.

Under the criteria of maximizing the expected utility of terminal wealth, most of the literature mentioned above are based on expected value premium principle due to its simplicity and popularity in practice. Sun et al. [14] consider the optimal investment-reinsurance strategies under variance premium principle. Zeng et al. [17] and $\mathrm{Gu}$ et al. [18] investigate the optimal proportional reinsurance-investment problem for an insurer with mispricing, model ambiguity with mean-reversion under expected value premium principle. Motivated by these papers, both the insurance and reinsurance premium payments are calculated by using the variance premium principle in this paper.

The rest of the paper is organized as follows. In Section 2, we provide the financial market and the insurance model. In Section 3, the optimal robust proportional reinsurance-investment problem is established. Optimal proportional reinsurance-investment strategies and their corresponding optimal value functions are given in Section 4.

\section{Economy and Assumption}

In this section, we formulate a continuous-time financial model where the insurers can trade in the financial market and in the insurance market with no taxes or fees. Let $\left(\Omega, \mathcal{F},\left\{\mathcal{F}_{t}, 0 \leq t \leq T\right\}, P\right)$ be a probability space, in which $\Omega$ is the state space and $\mathcal{F}$ is a $\sigma$-algebra on $\Omega . T>0$ is a fixed constant, representing the time horizon, $\left\{\mathcal{F}_{t}\right\}_{t \geq 0}$ is a filtration, which describes the flow 
of information over time, the $\sigma$-algebra $\left\{\mathcal{F}_{t}\right\}$ describes the information available up to time $t$, and $\left\{\mathcal{F}_{t}\right\}_{t \geq 0}$ satisfies the usual condition (it contains all P-null sets and is right continuous). We denote $P$ as a reference measure and suppose that all stochastic processes given in the following are assumed to be adapted on this space.

\subsection{Surplus Process}

We assume that the insurer's surplus is given by the classical Cramér-Lunderberg risk model (without reinsurance and investment), the insurer's surplus $\mathrm{R}$ is given by

$$
\mathrm{d} R(t)=c \mathrm{~d} t-d \sum_{i=1}^{N_{t}} Y_{i}+\sigma_{1} \mathrm{~d} B(t),
$$

where $c$ is the premium rate, the claim arrival process $\left\{N_{t}\right\}_{t>0}$ is a Poisson process with constant intensity $\lambda>0$ and the random variables $Y_{i}, i=1,2, \cdots$ are i.i.d claim sizes independent of $N_{t}$. We let $F(y)$ denote the claim size distribution with finite first-order moment $m_{1}$ and second-order moment $m_{2}$. The stochastic process $\{B(t), 0 \leq t \leq T\}$ is a standard Brownian motion independent of $N$, representing the diffusion risk of the surplus process. The premium rate $c$ is assumed to be calculated via the expected value principle, i.e.,

$$
c=E_{P}\left[\sum_{i=1}^{N(t)} Y_{i}\right]+\theta_{0} \operatorname{Var}\left[\sum_{i=1}^{N(t)} Y_{i}\right]=\lambda\left(m_{1}+\theta_{0} m_{2}\right),
$$

where $\theta_{0}$ is the insurer's safety loading.

In this paper, the insurer can purchase proportional reinsurance or acquire new business to adjust the exposure to insurance risk. The proportional reinsurance/new business level is associated with the risk exposure $q(t)$ at time $t$. When $q(t) \in[0,1]$, it means the insurer purchases proportional reinsurance. In this case, for each claim, the insurer only pays its $q(t) Y$, while the reinsurer pays the rest $(1-q(t)) Y$ for each claim. When $q(t) \in[1, \infty]$, it means the insurer acquires new business from the other insurers as a reinsurer. Then, the aggregate reinsurance premium under the variance principle takes the form

$$
E_{P}\left[\sum_{i=1}^{N(t)}\left(Y_{i}-q Y_{i}\right)\right]+\theta_{1} \operatorname{Var}\left[\sum_{i=1}^{N(t)}\left(Y_{i}-q Y_{i}\right)\right]=\lambda\left[(1-q) m_{1}+\theta_{1}(1-q)^{2} m_{2}\right] t,
$$

with $\theta_{1}>0$. Thus, the surplus process of the insurer after taking into account reinsurance is governed by

$$
\begin{aligned}
\mathrm{d} R(t) & =\left[q(t) m_{1}+\left[\theta_{0}-\theta_{1}(1-q(t))^{2}\right] m_{2}\right] \lambda \mathrm{d} t-q(t) \mathrm{d} \sum_{i=1}^{N_{t}} Y_{i} \\
& =\left[q(t) m_{1}+\left[\theta_{0}-\theta_{1}(1-q(t))^{2}\right] m_{2}\right] \lambda \mathrm{d} t-q(t) \int_{R^{\prime}} y(t) N(\mathrm{~d} t, \mathrm{~d} y),
\end{aligned}
$$

where $N(\cdot, \cdot)$ defined on $\Omega \times[0, T] \times[0, \infty)$ is a Poisson random measures used to represent the compound Poisson process $\sum_{i=1}^{N(t)} Y_{i}$ as 


$$
\sum_{i=1}^{N(t)} Y_{i}=\int_{0}^{t} \int_{R^{+}} y N(\mathrm{~d} t, \mathrm{~d} y)
$$

If we denote $v(\mathrm{~d} t, \mathrm{~d} y)=\lambda \mathrm{d} t \mathrm{~d} F(y)$, then

$$
E\left[\sum_{i=1}^{N(t)} Y_{i}\right]=\int_{0}^{t} \int_{R^{+}} y v(\mathrm{~d} t, \mathrm{~d} y)
$$

and $v(\mathrm{~d} t, \mathrm{~d} y)$ is the compensator of the random measure $N(\cdot, \cdot)$. Thus, the compensated measures $\tilde{N}(\cdot, \cdot)=N(\cdot, \cdot)-v(\cdot, \cdot)$ is related to the compound Poisson process $\sum_{i=1}^{N(t)} Y_{i}$ as follows

$$
\int_{0}^{t} \int_{R^{+}} y \tilde{N}(\mathrm{~d} t, \mathrm{~d} y)=\sum_{i=1}^{N(t)} Y_{i}-E\left[\sum_{i=1}^{N(t)} Y_{i}\right], \forall t \in[0, T]
$$

\subsection{Financial Market}

We assume that financial market consists of one risk-free asset, and a pair of stocks with mispricing (two price processes for the same asset on two distinct stock markets). We assume for the moment that the price of risk-free asset $P_{0}$ is given by

$$
\mathrm{d} P_{0}(t)=r P_{0}(t) \mathrm{d} t, P_{0}(0)=p_{0},
$$

where constant $r>0$ is the risk-free interest rate. The price process of the market index is expressed as

$$
\frac{\mathrm{d} P_{m}(t)}{P_{m}(t)}=\left(r+\mu_{m}\right) \mathrm{d} t+\sigma_{m} \mathrm{~d} Z_{m}(t),
$$

where the market risk premium $\mu_{m}$, the market volatility $\sigma_{m}$ are positive constants, and $Z_{m}(t)$ is a standard Brownian motion on $(\Omega, \mathcal{F}, P)$. The two mispriced price processes are modeled as a pair of stocks $P_{1}$ and $P_{2}$ which are coupled via the pricing error

$$
X(t):=\ln \frac{P_{1}(t)}{P_{2}(t)} .
$$

We assume the vector $\left(P_{1}, P_{2}\right)$ solves the system of stochastic differential equations

$$
\begin{aligned}
& \frac{\mathrm{d} P_{1}(t)}{P_{1}(t)}=(r+a(t)) \mathrm{d} t+\sigma \mathrm{d} Z(t)+b \mathrm{~d} Z_{1}(t)-\lambda_{1} X(t) \mathrm{d} t, \quad P_{1}(0)=p_{10}, \\
& \frac{\mathrm{d} P_{2}(t)}{P_{2}(t)}=(r+a(t)) \mathrm{d} t+\sigma \mathrm{d} Z(t)+b \mathrm{~d} Z_{2}(t)+\lambda_{2} X(t) \mathrm{d} t, \quad P_{2}(0)=p_{20},
\end{aligned}
$$

in which $\lambda_{1}, \lambda_{2}, \sigma, b$ are constant parameters. And we assume the premium for the common risk part $\sigma \mathrm{d} Z(t)$ is the observed mean-reverting process $a(t)$ whose dynamics are given by

$$
\mathrm{d} a(t)=\kappa(\theta-a(t)) \mathrm{d} t+\sigma_{a} \mathrm{~d} Z_{a}(t), \quad a(0)=a_{0},
$$

where $\kappa$ is the long-run mean of the risk premium, and $\theta$ is the degree of 
mean reversion (or mean-reversion rate). The term $\lambda_{i} X(t) \mathrm{d} t$ shows the effect of mispricing on the ith stock's price. The term $\sigma \mathrm{d} Z(t)+b \mathrm{~d} Z_{i}(t)$ describe the idiosyncratic risk of stock $i, \sigma \mathrm{d} Z(t)$ describes the common risk, while the individual risk $b \mathrm{~d} Z_{i}(t)$ is generated by the asset $i, i=1,2$. The term $\lambda_{i} X(t) \mathrm{d} t$ shows the effect of miscricing on $i$ th stock's price via the pricing error $X(t)$ defined above. We assume that all standard Brownian motions $Z(t), Z_{1}(t)$, $Z_{2}(t)$ and $Z_{a}(t)$ are independent of each other and all are independent of $N(\mathrm{~d} t, \mathrm{~d} y)$. Based on (11) and (12), ordinary stochastic calculus implies that the dynamics of the pricing error $X(t)$ is given by the following equation

$$
\begin{aligned}
& \mathrm{d} X(t)=-\left(\lambda_{1}+\lambda_{2}\right) X(t) \mathrm{d} t+b \mathrm{~d} Z_{1}(t)-b \mathrm{~d} Z_{2}(t), \quad X(0)=x_{0} . \\
& \mathrm{d} X(t)=-\left(\lambda_{1}+\lambda_{2}\right) X(t) \mathrm{d} t+b \mathrm{~d} Z_{1}(t)-b \mathrm{~d} Z_{2}(t), \quad X(0)=x_{0} .
\end{aligned}
$$

Thus Equation (14) shows that $X$ is also a MR process. Note that $\rho$ is the correlation coefficient between the Brownian motions $Z(t)$ and $B(t)$, and $\rho_{0}$ is the correlation coefficient between $Z_{m}(t)$ and $Z_{a}(t)$. Moreover, there exist Brownian motions $\hat{B}(t)$ and $Z_{0}(t)$ satisfying the following equations:, where. Moreover, we assume all standard Brownian motions $\left\{Z_{m}(t)\right\},\{B(t)\}$, $\{\hat{B}(t)\},\left\{Z_{1}(t)\right\},\left\{Z_{2}(t)\right\}$ and $\left\{Z_{0}(t)\right\}$ are independent each other and all are independent of $N(\mathrm{~d} y, \mathrm{~d} t)$. To capture the features of mispricing, we assume $\lambda_{1}+\lambda_{2}>0$.

In addition to reinsurance, the insurer can invest in our financial market. Thus we denote by $u(t)=\left(\ell_{m}(t), \ell_{1}(t), \ell_{2}(t)\right)$ the corresponding investment strategy, where $\ell_{m}(t)$ shows the amount of the wealth invested in the market index, $\ell_{1}(t)$, and $\ell_{2}(t)$ denote the amounts invested in the two stocks, respectively, hence the remainder, $R(t)-\ell_{m}(t)-\ell_{1}(t)-\ell_{2}(t)$ is invested in the risk-free asset.

\subsection{Wealth Process}

We denote the whole reinsurance-investment strategy by $\ell:=\{\ell(t), 0 \leq t \leq T\}=\{q(t), u(t), t \in[0, T]\}$. As a result of adopting the strategy $\ell$, the insurer's corresponding reserve $\left\{W^{\ell}(t)\right\}_{0 \leq t \leq T}$ satisfies the following stochastic dynamics:

$$
\begin{aligned}
& \mathrm{d} W^{\ell}(t) \\
&=\left[W^{\ell}(t) r+\ell_{m}(t) \mu_{m}+a(t)\left(\ell_{1}(t)+\ell_{2}(t)\right)+X(t)\left(\lambda_{2} \ell_{2}(t)-\lambda_{1} \ell_{1}(t)\right)\right] \mathrm{d} t \\
&+\sigma_{1} \mathrm{~d} B(t)+\sigma\left(\ell_{1}(t)+\ell_{2}(t)\right)(\rho \mathrm{d} B(t)+\hat{\rho} \mathrm{d} \hat{B}(t))+b \ell_{1}(t) \mathrm{d} Z_{1}(t) \\
&+b \ell_{2}(t) \mathrm{d} Z_{2}(t)+\ell_{m}(t) \sigma_{m} \mathrm{~d} Z_{m}(t)+\left[q(t) m_{1}+\left[\theta_{0}-\theta_{1}(1-q(t))^{2}\right] m_{2}\right] \lambda \mathrm{d} t \\
&-q(t) \int_{R^{+}} y(t) N(\mathrm{~d} t, \mathrm{~d} y),
\end{aligned}
$$

where $W^{\ell}(0)=w_{0}$ and $w_{0}$ is the insurer's initial wealth.

\section{Robust Problem with Mispricing}

To derive explicit results, we need to make some assumptions regarding the am- 
biguity-averse insurer's utility. Suppose that the insurer has exponential utility function, i.e.

$$
U(x)=-\frac{1}{\gamma} \exp \{-\gamma x\}
$$

where $\gamma>0$ is a constant representing the coefficient of absolute risk aversion. The insurer is assumed to be ambiguity-neutral with objective function

$$
\max _{\ell \in \Pi_{0}} E_{0}^{P}\left[U\left(W^{\ell}(T)\right)\right]=\max _{\ell \in \Pi_{0}} E_{0}^{P}\left[-\frac{1}{\gamma} \exp \left\{-\gamma W^{\ell}(T)\right\} .\right.
$$

where $\Pi_{0}$ is the set of admissible strategies for an ambiguity-neutral insurer in a given market and $E_{t}^{P}[\cdot]=E^{P}\left[\cdot \mid \mathcal{F}_{t}\right]$ represents the conditional expectation under the probability measure $P$.

Definition 3.1. A strategy $\ell=\left\{q(t), \ell_{m}(t), \ell_{1}(t), \ell_{2}(t)\right\}_{t \in[0, T]}$ is said to be admissible, if

1) $\forall t \in[0, T], q(t) \in[0, \infty)$;

2) $\ell$ is predictable with respect to $\mathcal{F}_{t}$ and $E^{Q^{*}}\left[\int_{0}^{T}\|v(t)\|^{2} \mathrm{~d} t\right]<\infty$, where $\|v(t)\|^{2}=q(t)^{2}+\ell_{m}^{2}(t)+\ell_{1}(t)^{2}+\ell_{2}(t)^{2} ;$

3) $\forall(t, w, x, a) \in[0, T] \times R \times R \times R$, Equation (5) has a unique solution $\left\{W^{\ell}(t)\right\}_{t \in[0, T]}$ with $E_{t, w, x, a}^{Q^{*}}\left[U\left(W^{\ell}(t)\right)\right]<\infty$, where $Q^{*}$ is the chosen model to describe the worst case and $E_{t, w}^{Q^{*}}[\cdot]=E^{Q^{*}}\left[\cdot \mid W^{\ell}(t)=w\right]$.

Denote by $\ell$ the set of all admissible strategies.

In order to incorporate the ambiguity, we assume that the insurer does not have full confidence in the reference probability $P$. She uses the probability $P$ as her reference probability for the wealth process, but takes some alternative models at the same time. Every alternative model is characterized by another stochastic process and the associated probability measure $Q$, which is equivalent to the reference measure $P$. We denote this class of probability measures by $\mathcal{Q}$ : $\mathcal{Q}:=\{Q: Q \sim P\}$.

The change of measure from $P$ to $Q$ can be defined by its Randon-Nikodym derivative, i.e., there exists a progressively measurable process $\epsilon=(\varphi(s), \phi(t))$, such that every $Q$ in $\mathcal{Q}$ satisfies

$$
\frac{\mathrm{d} Q}{\mathrm{~d} P}=\xi(T)
$$

where

$$
\begin{aligned}
\xi(t)= & \exp \left\{-\int_{0}^{t} \varphi(t) \mathrm{d} \mathcal{Z}(t)-\frac{1}{2} \int_{0}^{t}\|\varphi(t)\|^{2} \mathrm{~d} t-\int_{0}^{t} h(t) \mathrm{d} B(t)-\frac{1}{2} \int_{0}^{t} h^{2}(t) \mathrm{d} t\right. \\
& \left.+\int_{0}^{t} \int_{0}^{\infty} \ln \phi(t) N(\mathrm{~d} t, \mathrm{~d} y)+\int_{0}^{t} \int_{0}^{\infty}(1-\phi(t)) v(\mathrm{~d} y, \mathrm{~d} t)\right\},
\end{aligned}
$$

where $\varphi(t)=\left(h_{m}(t), \hat{h}(t), h_{1}(t), h_{2}(t), h_{0}(t)\right)$, $\|\varphi(t)\|^{2}=h_{m}^{2}(t)+\hat{h}^{2}(t)+h_{1}^{2}(t)+h_{2}^{2}(t)+h_{0}^{2}(t)$ and 
$\mathrm{d} \mathcal{Z}(t)=\left(\mathrm{d} Z_{m}(t), \mathrm{d} \hat{B}(t), \mathrm{d} Z_{1}(t), \mathrm{d} Z_{2}(t), \mathrm{d} Z_{0}(t)\right)$. According to Girsanov's theorem ( $\varnothing \mathrm{ksendal}[19])$, under the alternative measure $Q$, the stochastic process $\left\{Z_{0}^{Q}(t)\right\},\left\{Z_{1}^{Q}(t)\right\},\left\{Z_{2}^{Q}(t)\right\},\left\{Z_{m}^{Q}(t)\right\},\left\{B^{Q}(t)\right\}$ and $\left\{B^{Q}(t)\right\}$ are standard Brownian motions, where

$$
\begin{aligned}
& \mathrm{d} Z_{0}^{Q}(t)=\mathrm{d} Z_{0}(t)+h_{0}(t) \mathrm{d} t, \mathrm{~d} Z_{1}^{Q}(t)=\mathrm{d} Z_{1}(t)+h_{1}(t) \mathrm{d} t, \\
& \mathrm{~d} Z_{2}^{Q}(t)=\mathrm{d} Z_{2}(t)+h_{2}(t) \mathrm{d} t, \mathrm{~d} Z_{m}^{Q}(t)=\mathrm{d} Z_{m}(t)+h_{m}(t) \mathrm{d} t, \\
& \mathrm{~d} B^{Q}(t)=\mathrm{d} B(t)+h(t) \mathrm{d} t, \mathrm{~d} \hat{B}^{Q}(t)=\mathrm{d} \hat{B}(t)+\hat{h}(t) \mathrm{d} t .
\end{aligned}
$$

Moreover, the intensity of the Poisson process becomes $\lambda \phi(t)$, that is,

$$
\tilde{N}^{Q}(\mathrm{~d} t, \mathrm{~d} y)=N(\mathrm{~d} t, \mathrm{~d} y)-\lambda^{Q} \mathrm{~d} F(y) \mathrm{d} t=N(\mathrm{~d} t, \mathrm{~d} y)-\lambda \phi(t) \mathrm{d} F(y) \mathrm{d} t,
$$

is a martingale. For tractability and ease of interpretation, the distribution of the claim $Y$ is assumed to be known, and is restricted to be identical under $P$ and $Q$. Thus, the dynamics of the wealth process under $Q$ is

$$
\begin{aligned}
\mathrm{d} W^{\ell}(t)= & {\left[W^{\ell}(t) r+\ell_{m}(t) \mu_{m}+a(t)\left(\ell_{1}(t)+\ell_{2}(t)\right)+X(t)\left(\lambda_{2} \ell_{2}(t)-\lambda_{1} \ell_{1}(t)\right)\right.} \\
& -\ell_{m}(t) \sigma_{m} h_{m}(t)-\sigma\left(\ell_{1}(t)+\ell_{2}(t)\right)(\rho h(t)+\hat{\rho} \hat{h}(t))-b\left(\ell_{1}(t) h_{1}(t)\right. \\
& \left.\left.+\ell_{2}(t) h_{2}(t)\right)\right] \mathrm{d} t-\sigma_{1} h(t) \mathrm{d} t+\ell_{m} \sigma_{m} \mathrm{~d} Z_{m}^{Q}(t)+\ell_{1}(t) b \mathrm{~d} Z_{1}^{Q}(t) \\
& +\ell_{2}(t) b d Z_{2}^{Q}(t)+\sigma_{1} \mathrm{~d} B^{Q}(t)+\sigma\left(\ell_{1}(t)+\ell_{2}(t)\right)(\rho \mathrm{d} B(t)+\hat{\rho} \mathrm{d} \hat{B}(t)) \\
& +\left[q(t) m_{1}+\left[\theta_{0}-\theta_{1}(1-q(t))^{2}\right] m_{2}\right] \lambda \mathrm{d} t \\
& -q(t) \int_{R^{+}} y\left(\tilde{N}^{Q}(\mathrm{~d} t, \mathrm{~d} y)+\lambda \phi(t) \mathrm{d} F(y) \mathrm{d} t\right),
\end{aligned}
$$

Meanwhile, the dynamics of the mispricing error under $Q$ can be given by

$$
\mathrm{d} X(t)=\left[-\left(\lambda_{1}+\lambda_{2}\right) X(t)-b h_{1}(t)+b h_{2}(t)\right] \mathrm{d} t+b \mathrm{~d} Z_{1}^{Q}(t)-b \mathrm{~d} Z_{2}^{Q}(t),
$$

and (13) can be modified to

$$
\begin{aligned}
\mathrm{d} a(t)= & {\left[\kappa(\theta-a(t))-h_{m}(t) \rho_{0} \sigma_{a}-h_{0}(t) \hat{\rho}_{0} \sigma_{a}\right] \mathrm{d} t } \\
& +\sigma_{a} \rho_{0} \mathrm{~d} Z_{m}^{Q}(t)+\hat{\rho}_{0} \sigma_{a} \mathrm{~d} Z_{0}^{Q}(t) .
\end{aligned}
$$

Following from Maenhout [10], Gu et al. [16] and Zeng et al. [20], we show that the increase in relative entropy from $t$ to $t+\mathrm{d} t$ equals

$$
\begin{aligned}
& \frac{1}{2}\left(h_{m}^{2}(t)+h^{2}(t)+\hat{h}^{2}(t)+h_{1}^{2}(t)+h_{2}^{2}(t)+h_{0}^{2}(t)\right) \mathrm{d} t \\
& +\lambda(\phi(t) \ln \phi(t)-\phi(t)+1) \mathrm{d} t .
\end{aligned}
$$

Denote that

$$
J(t, w, x, a)=\operatorname{supinf}_{\ell \in \Pi} E_{Q \in \mathcal{Q}}^{Q}\left\{-\frac{1}{\gamma} \mathrm{e}^{-\gamma w(T)}+\int_{t}^{T}\left(\frac{R_{1}(s)}{\psi_{1}(s)}+\frac{R_{2}(s)}{\psi_{2}(s)}+\frac{R_{3}(s)}{\psi_{3}(s)}\right) \mathrm{d} s\right\},
$$

where $R_{1}(t)=\varphi^{2}(s)=\frac{1}{2}\left(h_{m}^{2}(t)+\hat{h}^{2}(t)+h_{1}^{2}(t)+h_{2}^{2}(t)+h_{0}^{2}(t)\right), \quad R_{2}(t)=\frac{1}{2} h^{2}(t)$, $R_{3}(t)=\lambda(\phi(t) \ln \phi(t)-\phi(t)+1)$. For convenience, similar to Maenhout [10] and $\mathrm{Gu}$ et al. [16], we assume that $\psi_{1}(t), \psi_{2}(t)$ and $\psi_{3}(t)$ are non-negative and state-dependent functions which are inversely proportional to the value 
function:

$$
\psi_{1}(s)=-\frac{\alpha_{1}}{\gamma J(s, w, x, a)}, \psi_{2}(s)=-\frac{\alpha_{2}}{\gamma J(s, w, x, a)}, \psi_{3}(s)=-\frac{\alpha_{3}}{\gamma J(s, w, x, a)},
$$

where $\alpha_{1} \geq 0, \alpha_{2} \geq 0$ and $\alpha_{3} \geq 0$ represent the insurer's ambiguity aversion levels to the diffusion modeling and jump modeling risk.

Next we aim to derive the explicit solution to the HJB Equation (22) with preference parameter (27).

\section{Optimal Robust Investment and Reinsurance Strategy}

The purpose of this section is to find the optimal investment strategy $\left(\pi_{m}(t), \pi_{1}(t), \pi_{2}(t)\right)$ and the optimal proportional reinsurance strategy $q(t)$ under the worst-case scenario. According to the principle of dynamic programming, the robust Hamilton-Jacobi-Bellmann (HJB) equation established by Anderson et al. [21] to express the value function (26), can be derived as

$$
\begin{aligned}
& \operatorname{supinf}_{\ell \in \Pi(\varphi, \phi)}\left\{J_{t}+J_{w}\left[w r+a(t)\left(\ell_{1}(t)+\ell_{2}(t)\right)-\sigma(\rho h(t)+\hat{\rho} \hat{h}(t))\left(\ell_{1}(t)+\ell_{2}(t)\right)\right.\right. \\
& -\sigma_{1} h(t)+x\left(\lambda_{2} \ell_{2}(t)-\lambda_{1} \ell_{1}(t)\right)-b\left(\ell_{1}(t) h_{1}(t)+\ell_{2}(t) h_{2}(t)\right)+\ell_{m}(t) \mu_{m} \\
& \left.-\ell_{m}(t) \sigma_{m} h_{m}(t)\right]+\frac{1}{2} J_{w w}\left(\sigma^{2}\left(\ell_{1}(t)+\ell_{2}(t)\right)^{2}+b^{2} \ell_{1}^{2}(t)+b^{2} \ell_{2}^{2}(t)+\ell_{m}^{2}(t) \sigma_{m}^{2}\right. \\
& \left.+\sigma_{1}^{2}+2 \sigma_{1} \sigma \rho\left(\ell_{1}(t)+\ell_{2}(t)\right)\right)+b^{2} J_{x x}+\frac{1}{2} \sigma_{a}^{2} J_{a a}+J_{w x} b^{2}\left(\ell_{1}(t)-\ell_{2}(t)\right) \\
& +J_{w a} \sigma_{a} \rho_{0} \ell_{m}(t) \sigma_{m}+J_{a}\left(\kappa(\theta-a(t))-\sigma_{a} \rho_{0} h_{m}(t)-\sigma_{a} \hat{\rho} h_{0}(t)\right) \\
& +J_{w}\left[q(t) m_{1}+\left[\theta_{0}-\theta_{1}(1-q(t))^{2}\right] m_{2}\right] \lambda+J_{x}\left(b h_{2}(t)-b h_{1}(t)-\left(\lambda_{1}+\lambda_{2}\right) x\right) \\
& -\frac{\gamma J \lambda}{\alpha_{3}}(\phi(t) \ln \phi(t)-\phi(t)+1)-\frac{\gamma J}{\alpha_{1}} \frac{1}{2}\|\varphi(t)\|^{2}-\frac{\gamma J}{\alpha_{2}} \frac{1}{2} h(t)^{2} \\
& \left.+\phi(t) \lambda E^{Q}[J(t, w-q(t) Y, x, a)-J(t, w, x, a)]\right\}=0
\end{aligned}
$$

with the boundary condition $J(T, w, x, a)=-\frac{1}{\gamma} \exp \{-\gamma w\}$, and $J_{t}, J_{w}, J_{a}$, $J_{x}, J_{w w}, J_{w x}, J_{x x}$ and $J_{a a}$ represent the value function's partial derivative w.r.t the corresponding variables.

In order to obtain the solution $J$ of (28), we conjecture that $J(t, w, x, a)$ has the ansatz form

$$
\begin{aligned}
J(t, w, x, a)= & -\frac{1}{\gamma} \exp \left\{-\gamma\left[P(t) w+\frac{1}{2} A_{2}(t) a^{2}+A_{1}(t) a+A_{0}(t)\right.\right. \\
& \left.\left.+\frac{1}{2} B_{1}(t) x^{2}+B_{2}(t) x+B_{3}(t) a x\right]\right\}
\end{aligned}
$$

with the boundary condition $P(T)=1$,

$A_{0}(T)=A_{1}(T)=A_{2}(T)=B_{1}(T)=B_{2}(T)=0$. A direct calculation yields partial derivatives 


$$
\begin{aligned}
& J_{t}=-\gamma\left(P^{\prime}(t) w+\frac{1}{2} A_{2}^{\prime}(t) a^{2}+A_{1}^{\prime}(t) a+A_{0}^{\prime}(t)+\frac{1}{2} B_{1}^{\prime}(t) x^{2}+B_{2}^{\prime}(t) x+B_{3}^{\prime}(t) a x\right) J, \\
& J_{a}=-\gamma\left(A_{2}(t) a+A_{1}(t)+B_{2}(t) x\right) J, J_{w}=-\gamma P(t) J, J_{w w}=\gamma^{2} P^{2}(t) J, \\
& J_{x}=-\gamma\left(B_{1}(t) x+B_{2}(t)+B_{3}(t) a\right) J, J_{w x}=\gamma^{2} P(t)\left(B_{1}(x)+B_{2}(t)+B_{3}(t) a\right) J, \\
& J_{x x}=\left[-\gamma B_{1}(t)+\gamma^{2}\left(B_{1}(t) x+B_{2}(t)+B_{3}(t) a\right)^{2}\right] J, \\
& J_{a a}=\gamma\left(\left(A_{2}(t) a+A_{1}(t)+B_{2} x\right)^{2}-A_{2}(t)\right) J, \\
& J_{w a}=\gamma^{2} P(t)\left(A_{2}(t) a+A_{1}(t)+B_{2}(t) x\right) J, \\
& \frac{J_{w}}{J_{w w}}=-\frac{1}{\gamma P(t)}, \frac{J_{w x}}{J_{w w}}=\frac{B_{1}(x)+B_{2}(t)+B_{3}(t) a}{P(t)} \\
& J(t, w-q Y, x, a)-J(t, w, x, a)=J(t, w, x, a)(\exp \{\gamma P(t) q(t) Y\}-1), \\
& \text { where } J=J(t, w, x, a) .
\end{aligned}
$$

Substituting (30) back into Equation (28) and according to the first-order conditions for $\left(h_{m}(t), h(t), h_{1}(t), h_{2}(t), h_{0}(t), \phi\right)$, we can obtain the minimum point $\left(h_{m}^{*}(t), h^{*}(t), h_{1}^{*}(t), h_{2}^{*}(t), h_{0}^{*}(t), \phi^{*}\right)$ given by

$$
\begin{aligned}
h_{m}^{*}(t) & =-\frac{\alpha_{1}}{\gamma J}\left(J_{w} \pi_{m} \sigma_{m}+J_{a} \sigma_{a} \rho_{0}\right) \\
& =\alpha_{1}\left(\pi_{m}(t) \sigma_{m} P(t)+\sigma_{a} \rho_{0}\left(A_{2}(t) a+A_{1}(t)+B_{3}(t)\right)\right) ; \\
\hat{h}^{*}(t) & =-\frac{\alpha_{1}}{\gamma J}\left(\pi_{1}(t)+\pi_{2}(t)\right) \sigma \hat{\rho} J_{w}=\alpha_{1} \sigma \hat{\rho} P(t)\left(\pi_{1}(t)+\pi_{2}(t)\right) ; \\
h_{0}^{*}(t) & =-\frac{\alpha_{1}}{\gamma J} \sigma_{a} J_{a} \hat{\rho}_{0}=\alpha_{1} \sigma_{a} \hat{\rho}_{0}\left(A_{2}(t) a+A_{1}(t)-B_{3}(t) a\right) ; \\
h_{1}^{*}(t) & =-\frac{\alpha_{1}}{\gamma J}\left(b \pi_{1}(t) J_{w}+b J_{x}\right)=\alpha_{1} b\left(\pi_{1}(t) P(t)+B_{1}(t) x+A_{2}(t)+B_{3}(t) a\right) ; \\
h_{2}^{*}(t) & =-\frac{\alpha_{1}}{\gamma J}\left(b \pi_{2}(t) J_{w}-b J_{x}\right)=\alpha_{1} b\left(\pi_{2}(t) P(t)-B_{1}(t) x-A_{2}(t)-B_{3}(t) a\right), \\
\phi^{*}(t) & =\exp \left\{\frac{\alpha_{3}}{\gamma} E^{P}\left[\mathrm{e}^{P(t) q(t) \gamma Y}-1\right]\right\} .
\end{aligned}
$$

We substitute (31) into the HJB Equation (28), and differentiating w.r.t. $q(t)$ implies

$$
\begin{aligned}
& \sup _{\ell \in \Pi}\left\{J_{t}+J_{w}\left[r w+a\left(\ell_{1}(t)+\ell_{2}(t)\right)+x\left(\lambda_{2} \ell_{2}(t)-\lambda_{1} \ell_{1}(t)\right)+\ell_{m}(t) \mu_{m}\right]\right. \\
& +J_{a} \kappa(\theta-a)+J_{w}\left[q(t) m_{1}+\left(\theta_{0}-\theta_{1}(1-q(t))^{2}\right) m_{2}\right] \lambda+J_{w x} b^{2} w\left(\ell_{1}(t)-\ell_{2}(t)\right) \\
& +b^{2} J_{x x}+\frac{1}{2} \sigma_{a}^{2} J_{a a}+\frac{1}{2} J_{w w} w^{2}\left(\ell_{m}^{2}(t) \sigma_{m}^{2}+\sigma^{2}\left(\ell_{1}(t)+\ell_{2}(t)\right)^{2}+b^{2} \ell_{1}^{2}(t)+b^{2} \ell_{2}^{2}(t)\right. \\
& \left.+\sigma_{1}^{2}+2 \sigma_{1} \sigma \rho\left(\ell_{1}(t)+\ell_{2}(t)\right)\right)-J_{x}\left(\lambda_{1}+\lambda_{2}\right) x+\frac{\gamma J}{\alpha_{3}}\left(\phi^{*}(t)-1\right) \lambda
\end{aligned}
$$




$$
\begin{aligned}
& +\frac{1}{2} \frac{\alpha_{1}}{\gamma J} w^{2} \sigma^{2}\left(\ell_{1}(t)+\ell_{2}(t)\right)^{2} J_{w}^{2}+J_{w a} \sigma_{a} \rho_{0} \ell_{m} \sigma_{m}+\frac{\alpha_{1}}{2 \gamma J}\left(w b \ell_{1}(t) J_{w}+b J_{x}\right)^{2} \\
& +\frac{\alpha_{1}}{2 \gamma J}\left(b \ell_{2}(t) J_{w}-b J_{x}\right)^{2}+\frac{\alpha_{1}}{2 \gamma J} \sigma_{a}^{2} J_{a}^{2} \hat{\rho}_{0}^{2} \frac{\alpha_{1} \sigma^{2} \hat{\rho}^{2} J_{w}^{2}\left(\ell_{1}(t)+\ell_{2}(t)\right)^{2}}{2 \gamma J} \\
& \left.+\frac{\alpha_{1}}{2 \gamma J}\left(J_{w} \ell_{m}(t) \sigma_{m}+J_{a} \sigma_{a} \rho_{0}\right)^{2}+\frac{\alpha_{2} J_{w}^{2}\left(\sigma_{1}+\left(\ell_{1}(t)+\ell_{2}(t)\right) \sigma \rho\right)^{2}}{2 \gamma J}\right\}=0 .
\end{aligned}
$$

Differentiating Equation (32) w.r.t. $q(t)$, we get the optimal reinsurance strategy $q^{*}(t)$ satisfies

$$
m_{1}+2 \theta_{1}(1-q(t)) m_{2}=\exp \left\{\frac{\alpha_{3}}{\gamma} E^{P}\left[\mathrm{e}^{P(t) q^{*}(t) \gamma Y}-1\right]\right\} E^{P}\left[Y \mathrm{e}^{P(t) q^{*}(t) \gamma Y}\right] .
$$

Using the result of $\mathrm{Gu}$ et al. [16], we get $q^{*}(t)>0$. If this were not true, then $\mathrm{e}^{P(t) q^{*}(t) \gamma Y}<1$ and

$\exp \left\{\frac{\alpha_{3}}{\gamma} E^{P}\left[\mathrm{e}^{P(t) q^{*}(t) \gamma Y}-1\right]\right\} E^{P}\left[Y \mathrm{e}^{P(t) q^{*}(t) \gamma Y}\right]<E^{P}[Y]<m_{1}+2 \theta_{1}(1-q(t)) m_{2}$. This would cause a contradiction with (33).

According to the first-order condition for $\ell_{m}(t), \ell_{1}(t)$ and $\ell_{2}(t)$, we have

$$
\begin{aligned}
& \ell_{m}^{*}(t) \\
& =\mathrm{e}^{-r(T-t)}\left[\frac{\mu_{m}}{\sigma_{m}\left(\alpha_{1}+\gamma\right)}-\frac{\sigma_{a} \rho_{0}}{\sigma_{m}}\left(A_{2}(t) a+A_{1}(t)+B_{3} x\right)\right],
\end{aligned}
$$

$\ell_{1}^{*}(t)$

$$
\begin{aligned}
& =\frac{1}{P(t)}\left[\left(\frac{1}{K} a\right)+\left(\frac{\lambda_{1}-\lambda_{2}}{2 K}\right) x-\left(B_{1}(t) x+B_{2}(t)+B_{3}(t) a\right)\right]-\frac{\left(\alpha_{2}+\gamma\right) \sigma_{1} \sigma \rho}{K}, \\
& \ell_{2}^{*}(t) \\
& =\frac{1}{P(t)}\left[\left(\frac{1}{K} a\right)+\left(\frac{\lambda_{1}-\lambda_{2}}{2 K}\right) x+\left(B_{1}(t) x+B(t)+B_{3}(t) a\right)\right]-\frac{\left(\alpha_{2}+\gamma\right) \sigma_{1} \sigma \rho}{K},
\end{aligned}
$$

where $K=2 \sigma^{2}\left(\gamma+\alpha_{1} \hat{\rho}^{2}+\alpha_{2} \rho^{2}\right)+b^{2}\left(\alpha_{1}+\gamma\right)$.

Inserting $q^{*}(t), \ell_{m}^{*}(t), \ell_{1}^{*}(t), \ell_{2}^{*}(t)$ into (32) and letting the coefficients of $w, a, x, a x, a^{2}$ and $x^{2}$ be zero, we get

$$
\begin{gathered}
-\gamma P^{\prime}(t)-\gamma r P(t)=0, \\
-\frac{1}{2} B_{1}^{\prime}(t)+\frac{1}{2} \sigma_{a}^{2}\left(\alpha_{1}+\gamma\right) \hat{\rho}_{0}^{2} B_{3}^{2}(t)-\frac{1}{4}\left(\frac{\left(\lambda_{1}-\lambda_{2}\right)^{2}}{K}+\frac{\left(\lambda_{1}+\lambda_{2}\right)^{2}}{\alpha_{1}+\gamma} b^{2}\right)=0, \\
-B_{2}^{\prime}(t)+\left(\alpha_{1}+\gamma\right) \sigma_{a}^{2} \hat{\rho}_{0}^{2} A_{1}(t) B_{3}(t)+\left(\frac{\mu_{m} \sigma_{a} \rho_{0}}{\sigma_{m}}-\kappa \theta\right) B_{3}(t) \\
+\frac{\alpha_{2} \sigma_{1} \sigma \rho P(t)\left(\lambda_{2}-\lambda_{1}\right)}{K}=0, \\
-\frac{1}{2} A_{2}^{\prime}(t)+\frac{1}{2} \sigma_{a}^{2}\left(\alpha_{1}+\gamma\right) \hat{\rho}_{0}^{2} A_{2}^{2}(t)+\kappa A_{2}(t)-\frac{1}{K}=0,
\end{gathered}
$$




$$
\begin{gathered}
-A_{1}^{\prime}(t)+\sigma_{a}^{2}\left(\alpha_{1}+\gamma\right) \hat{\rho}_{0}^{2} A_{1}^{2}(t) A_{2}(t)+\kappa A_{2}(t)+\left(\frac{\mu_{m} \sigma_{a} \rho_{0}}{\sigma_{m}}-\kappa \theta\right) A_{2}(t) \\
+\frac{2\left(\alpha_{2}+\gamma\right) \sigma_{1} \sigma P(t)}{K}-\frac{2 b^{2}\left(\alpha_{1}+\gamma\right) \gamma \sigma_{1} \sigma \rho P(t)}{K^{2}}=0 . \\
-B_{3}^{\prime}(t)+\kappa B_{3}(t)+\left(\alpha_{1}+\gamma\right) \sigma_{a}^{2} \hat{\rho}_{0}^{2} A^{1}(t)-\frac{\lambda_{2}-\lambda_{1}}{K}=0, \\
-A_{0}^{\prime}(t)+\frac{1}{2}\left(\alpha_{1}+\gamma\right) \sigma_{a}^{2} \hat{\rho}_{0}^{2} A_{1}^{2}(t)-b^{2} B_{1}(t)+\left(\frac{\mu_{m} \sigma_{a} \rho_{0}}{\sigma_{m}}-\kappa \theta\right) A_{1}(t)-\frac{1}{2} \sigma_{a}^{2} A_{2}(t) \\
-\frac{\mu_{m}^{2}}{2 \sigma_{m}^{2}\left(\alpha_{1}+\gamma\right)}+\frac{\left(\alpha_{2}+\gamma\right)\left(\gamma-\alpha_{2}\right) \sigma_{1}^{2} \sigma^{2} \rho^{2} P^{2}(t)}{K}+\frac{1}{2} \sigma_{1}^{2}\left(\alpha_{2}+\gamma\right) P^{2}(t) \\
-\left[q^{*}(t) m_{1}+\left(\theta_{0}-\theta_{1}\left(1-q^{*}(t)\right)^{2}\right) m_{2}\right] \lambda P(t)+\frac{\lambda}{\alpha_{3}}\left(\phi^{*}(t)-1\right)=0 .
\end{gathered}
$$

Taking into account the boundary conditions $P(T)=1$, $A_{0}(T)=A(T)=A_{2}(T)=B_{1}(T)=B_{2}(T)=0$, we know that

$$
P(t)=\mathrm{e}^{r(T-t)},
$$

$$
-\frac{\alpha_{2} \sigma_{1} \sigma \rho P(t)\left(\lambda_{2}-\lambda_{1}\right)}{K}(T-t),
$$

$$
B_{3}(t)=\mathrm{e}^{-\int_{t}^{T}\left(\kappa+\left(\alpha_{1}+\gamma\right) \hat{\sigma}_{a}^{2} \hat{\rho}_{0}^{2} A_{2}(s) \mathrm{ds}\right)} \times \int_{t}^{T} \frac{\lambda_{2}-\lambda_{1}}{K} \mathrm{e}^{\int_{s}^{T}\left(\kappa+\left(\alpha_{1}+\gamma\right) \hat{\sigma}_{a}^{2} \hat{\rho}_{0}^{2} A_{2}(u) \mathrm{d} u\right)},
$$

$A_{0}(t)=-\frac{\sigma_{a}^{2}\left(\alpha_{1}+\gamma\right) \hat{\rho}_{0}^{2}}{2} \int_{t}^{T} A_{1}^{2}(s) \mathrm{d} s+b^{2} \int_{t}^{T} B_{1}(s) \mathrm{d} s+\left(\frac{\mu_{m} \sigma_{a} \rho_{0}}{\sigma_{m}}-\kappa \theta\right) \int_{t}^{T} A_{1}(s) \mathrm{d} s$

$+\frac{\sigma_{a}^{2}}{2} \int_{t}^{T} A_{2}(s) \mathrm{d} s+\int_{t}^{T}\left(\frac{\mu_{m}^{2}}{2 \sigma_{m}^{2}\left(\alpha_{1}+\gamma\right)}-\frac{\left(\alpha_{2}+\gamma\right)\left(\gamma-\alpha_{2}\right) \sigma_{1}^{2} \sigma^{2} \rho^{2} P^{2}(s)}{K}\right.$

$\left.-\frac{1}{2} \sigma_{1}^{2}\left(\alpha_{2}+\gamma\right) P^{2}(s)\right) \mathrm{d} s+\int_{t}^{T}\left[q^{*}(s) m_{1}+\left(\theta_{0}-\theta_{1}\left(1-q^{*}(s)\right)^{2}\right) m_{2}\right] \lambda \mathrm{e}^{r(T-s)} \mathrm{d} s$ $-\int_{t}^{T} \frac{\lambda}{\alpha_{3}}\left(\phi^{*}(s)-1\right) \mathrm{d} s$,

$$
\begin{gathered}
A_{2}(t)=\frac{\mathrm{e}^{-\epsilon_{1}(T-t)}-\mathrm{e}^{-\epsilon_{2}(T-t)}}{\left(\frac{\mathrm{e}^{-\epsilon_{2}(T-t)}}{\epsilon_{2}}-\frac{\mathrm{e}^{-\epsilon_{1}(T-t)}}{\epsilon_{1}}\right)\left(\alpha_{1}+\gamma\right) \hat{\rho}_{0}^{2} \sigma_{a}^{2}}, \\
A_{1}(t)=-\mathrm{e}^{-\int_{t}^{T}\left(\kappa+\left(\alpha_{1}+\gamma\right)\right) \hat{\rho}_{0}^{2} \sigma_{a}^{2} A_{2}(s) \mathrm{d} s} \int_{t}^{T}\left(\frac{\mu_{m} \sigma_{a} \rho_{0}}{\sigma_{m}}-\kappa \theta\right) A_{2}(s) \times \mathrm{e}^{\int_{s}^{T}\left(\kappa+\left(\alpha_{1}+\gamma\right)\right) \hat{\rho}_{0}^{2} \sigma_{a}^{2} A_{2}(u) \mathrm{d} u} \mathrm{~d} s \\
-\mathrm{e}^{-\int_{t}^{T}\left(\kappa+\left(\alpha_{1}+\gamma\right)\right) \hat{\rho}_{0}^{2} \sigma_{a}^{2} A_{2}(s) \mathrm{d} s} \int_{t}^{T} \frac{2 \sigma_{1} \sigma \rho P(s)}{K}\left(\alpha_{2}+\gamma\right) \times \mathrm{e}^{\int_{s}^{T}\left(\kappa+\left(\alpha_{1}+\gamma\right)\right) \hat{\rho}_{0}^{2} \sigma_{a}^{2} A_{2}(u) \mathrm{d} u} \mathrm{~d} s \\
-\mathrm{e}^{-\int_{t}^{T}\left(\kappa+\left(\alpha_{1}+\gamma\right)\right) \hat{\rho}_{0}^{2} \sigma_{a}^{2} A_{2}(s) \mathrm{d} s} \int_{t}^{T} \frac{2 \sigma_{1} \sigma \rho P(s) b^{2}\left(\alpha_{1}+\gamma\right)}{K^{2}} \times \mathrm{e}^{\int_{s}^{T}\left(\kappa+\left(\alpha_{1}+\gamma\right)\right) \hat{\rho}_{0}^{2} \sigma_{a}^{2} A_{2}(u) \mathrm{d} u} \mathrm{~d} s,
\end{gathered}
$$


where $\epsilon_{1}=\kappa+\sqrt{\kappa^{2}+\frac{2 \sigma_{a}^{2}\left(\alpha_{1}+\gamma\right) \hat{\rho}_{0}^{2}}{K}}, \varepsilon_{2}=\epsilon-\sqrt{\kappa^{2}+\frac{2 \sigma_{a}^{2}\left(\alpha_{1}+\gamma\right) \hat{\rho}_{0}^{2}}{K}}$. Using the verification of Yi et al. [15], we have the following theorem.

Theorem 4.1. For the optimization problem (26), the value function is given by

$$
\begin{aligned}
J(t, w, x, a)= & -\frac{1}{\gamma} \exp \left\{-\gamma\left[P(t) w+\frac{1}{2} A_{2}(t) a^{2}+A_{1}(t) a+A_{0}(t)\right.\right. \\
& \left.\left.+\frac{1}{2} B_{1}(t) x^{2}+B_{2}(t) x+B_{3}(t) a x\right]\right\}
\end{aligned}
$$

where $P(t), A_{1}(t), A_{2}(t), A_{0}(t), B_{1}(t), B_{2}(t)$ and $B_{3}(t)$ are given by (38)-(43), and the corresponding optimal reinsurance-investment strategy is given by

$$
\ell=\left(q^{*}(t), \ell_{m}(t), \ell_{1}(t), \ell_{2}(t)\right),
$$

where $q^{*}(t)$ is given by Equation (33), and $\ell_{m}(t), \ell_{1}(t), \ell_{2}(t)$ are given by Equations (34)-(36).

If all the ambiguity-aversion coefficients equal 0, i.e. $\alpha_{1}=\alpha_{2}=\alpha_{3}=0$ in our model, the optimization problem degenerates into optimization problem (17) without ambiguity aversion. Similarly to Theorem 4.1, we have the following Proposition.

Proposition 4.1. For optimization problem (17), the value function is given by

$$
\begin{aligned}
J^{0}(t, w, x, a)= & -\frac{1}{\gamma} \exp \left\{-\gamma\left[\mathrm{e}^{r(T-t)} w+\frac{1}{2} A_{20}(t) a^{2}+A_{10}(t) a+A_{00}(t)\right.\right. \\
& \left.\left.+\frac{1}{2} B_{10}(t) x^{2}+B_{20}(t) x+B_{30}(t) a x\right]\right\}
\end{aligned}
$$

and the optimal strategy $\ell_{0}(t)=\left.\ell^{*}(t)\right|_{\alpha_{1}=\alpha_{2}=0}$, where $P(t), A_{10}(t), A_{20}(t)$, $A_{00}(t), B_{10}(t), B_{20}(t)$ and $B_{30}(t)$ are given by $(46)-(52)$

$$
\begin{gathered}
P(t)=\mathrm{e}^{r(T-t)}, \\
B_{10}(t)=\sigma_{a}^{2} \gamma \hat{\rho}_{0}^{2} \int_{t}^{T} B_{3}^{2}(s) \mathrm{d} s-\frac{T-t}{2}\left(\frac{\left(\lambda_{2}-\lambda_{1}\right)^{2}}{\left(2 \sigma^{2}+b^{2}\right) \gamma}+\frac{\left(\lambda_{1}+\lambda_{2}\right)^{2}}{\left(\alpha_{1}+\gamma\right) b^{2}}\right), \\
B_{20}(t)=\sigma_{a}^{2} \gamma \hat{\rho}_{0}^{2} \int_{t}^{T} A_{1}(s) B_{3}(s) \mathrm{d} s-\left(\frac{\mu_{m} \sigma_{a} \rho_{0}}{\sigma_{m}}-\kappa \theta\right) \int_{t}^{T} B_{3}(s) \mathrm{d} s, \\
B_{30}(t)=\mathrm{e}^{-\int_{t}^{T}\left(\kappa+\gamma \hat{\sigma}_{a}^{2} \hat{\rho}_{0}^{2} A_{2}(s) \mathrm{d} s\right)} \times \int_{t}^{T} \frac{\lambda_{2}-\lambda_{1}}{\left(2 \sigma^{2}+b^{2}\right) \gamma} \mathrm{e}^{\int_{s}^{T}\left(\kappa+\gamma \hat{\sigma}_{a}^{2} \hat{\rho}_{0} A_{2}(u) \mathrm{d} u\right)}, \\
+\frac{\sigma_{a}^{2} \gamma \hat{\rho}_{0}^{2}}{2} \int_{t}^{T} A_{1}^{2}(s) \mathrm{d} s+b^{2} \int_{t}^{T} B_{1}(s) \mathrm{d} s+\left(\frac{\mu_{m} \sigma_{a} \rho_{0}}{\sigma_{m}}-\kappa \theta\right) \int_{t}^{T} A_{1}(s) \mathrm{d} s \\
+\frac{\sigma_{a}^{2}}{2} \int_{t}^{T} A_{2}(s) \mathrm{d} s+\int_{t}^{T}\left(\frac{\mu_{m}^{2}}{2 \sigma_{m}^{2} \gamma}-\frac{\gamma \sigma_{1}^{2} \sigma^{2} \rho^{2} P^{2}(s)}{2 \sigma^{2}+b^{2}}-\frac{1}{2} \sigma_{1}^{2} \gamma P^{2}(s)\right) \mathrm{d} s \\
+\int_{t}^{T}\left[q^{*}(s) m_{1}+\left(\theta_{0}-\theta_{1}\left(1-q^{*}(s)\right)^{2}\right) m_{2}\right] \lambda \mathrm{e}^{r(T-s)} \mathrm{d} s \\
+\lambda E^{Q}\left[\mathrm{e}^{P(t) q_{0}(t) \gamma Y}-1\right],
\end{gathered}
$$




$$
\begin{gathered}
A_{20}(t)=\frac{\mathrm{e}^{-\epsilon_{1}(T-t)}-\mathrm{e}^{-\epsilon_{2}(T-t)}}{\left(\frac{\mathrm{e}^{-\epsilon_{2}(T-t)}}{\epsilon_{2}}-\frac{\mathrm{e}^{-\epsilon_{1}(T-t)}}{\epsilon_{1}}\right) \gamma \hat{\rho}_{0}^{2} \sigma_{a}^{2}}, \\
A_{10}(t)=-\mathrm{e}^{-\int_{t}^{T}(\kappa+\gamma) \hat{\rho}_{0}^{2} \sigma_{a}^{2} A_{2}(s) \mathrm{d} s} \int_{t}^{T}\left(\frac{\mu_{m} \sigma_{a} \rho_{0}}{\sigma_{m}}-\kappa \theta\right) A_{2}(s) \times \mathrm{e}^{\int_{s}^{T}(\kappa+\gamma) \hat{\rho}_{0}^{2} \sigma_{a}^{2} A_{2}(u) \mathrm{d} u} \mathrm{~d} s \\
-\mathrm{e}^{-\int_{t}^{T}(\kappa+\gamma) \hat{\rho}_{0}^{2} \sigma_{a}^{2} A_{2}(s) \mathrm{d} s} \int_{t}^{T} \frac{2 \sigma_{1} \sigma \rho P(s)}{\left(2 \sigma^{2}+b^{2}\right) \gamma} \gamma \times \mathrm{e}^{\int_{s}^{T}(\kappa+\gamma) \hat{\rho}_{0}^{2} \sigma_{a}^{2} A_{2}(u) \mathrm{d} u} \mathrm{~d} s \\
-\mathrm{e}^{-\int_{t}^{T}(\kappa+\gamma) \hat{\rho}_{0}^{2} \sigma_{a}^{2} A_{2}(s) \mathrm{d} s} \int_{t}^{T} \frac{2 \sigma_{1} \sigma \rho P(s) b^{2} \gamma}{\left(\left(2 \sigma^{2}+b^{2}\right) \gamma\right)^{2}} \times \mathrm{e}^{\int_{s}^{T}(\kappa+\gamma) \hat{\rho}_{0}^{2} \sigma_{a}^{2} A_{2}(u) \mathrm{d} u} \mathrm{~d} s, \\
\text { where } \epsilon_{1}=\kappa+\sqrt{\kappa^{2}+\frac{2 \sigma_{a}^{2} \hat{\rho}_{0}^{2}}{2 \sigma^{2}+b^{2}}}, \varepsilon_{2}=\epsilon-\sqrt{\kappa^{2}+\frac{2 \sigma_{a}^{2} \hat{\rho}_{0}^{2}}{2 \sigma^{2}+b^{2}}} .
\end{gathered}
$$

If the insurer only invests in the financial markets, and does not purchase reinsurance, the optimization problem (26) degenerates to investment only problem. With $q^{*}(t)=1$ in Theorem 4.1 , we easily derive the following proposition.

Proposition 4.2. For optimization problem (26), if only investment is discussed, the value function is given by

$$
\begin{aligned}
J(t, w, x, a)= & -\frac{1}{\gamma} \exp \left\{-\gamma\left[\mathrm{e}^{r(T-t)} w+\frac{1}{2} A_{2}(t) a^{2}+A_{1}(t) a+A_{01}(t)\right.\right. \\
& \left.\left.+\frac{1}{2} B_{1}(t) x^{2}+B_{2}(t) x+B_{3}(t) a x\right]\right\},
\end{aligned}
$$

where $A_{1}(t), A_{2}(t), B_{1}(t), B_{2}(t)$ and $B_{3}(t)$ are given by Theorem 4.1 , and

$$
\begin{aligned}
A_{01}(t)= & -\frac{\sigma_{a}^{2}\left(\alpha_{1}+\gamma\right) \hat{\rho}_{0}^{2}}{2} \int_{t}^{T} A_{1}^{2}(s) \mathrm{d} s+b^{2} \int_{t}^{T} B_{1}(s) \mathrm{d} s+\left(\frac{\mu_{m} \sigma_{a} \rho_{0}}{\sigma_{m}}-\kappa \theta\right) \int_{t}^{T} A_{1}(s) \mathrm{d} s \\
& +\frac{\sigma_{a}^{2}}{2} \int_{t}^{T} A_{2}(s) \mathrm{d} s+\int_{t}^{T}\left(\frac{\mu_{m}^{2}}{2 \sigma_{m}^{2}\left(\alpha_{1}+\gamma\right)}-\frac{\left(\alpha_{2}+\gamma\right)\left(\gamma-\alpha_{2}\right) \sigma_{1}^{2} \sigma^{2} \rho^{2} \mathrm{e}^{2 r(T-s)}}{K}\right. \\
& \left.-\frac{1}{2} \sigma_{1}^{2}\left(\alpha_{2}+\gamma\right) \mathrm{e}^{2 r(T-s)}\right) \mathrm{d} s+\int_{t}^{T}\left[q^{*}(s) m_{1}+\theta_{0} m_{2}\right] \lambda \mathrm{e}^{r(T-s)} \mathrm{d} s \\
& -\int_{t}^{T} \frac{\lambda}{\alpha_{3}}\left(\mathrm{e}^{\frac{\alpha_{3}}{\gamma} E^{Q}\left[\mathrm{e}^{r(T-s)} \gamma^{Y}-1\right]}-1\right) \mathrm{d} s .
\end{aligned}
$$

\section{Acknowledgements}

The research was supported by the National Natural Science Foundation of China (No.11501319), the Education Department of Shandong Province Science and Technology Plan Project (No. J15L105), the Natural Science Foundation of Shandong Province (No. ZR2015AL013) and the China Postdoctoral Science Foundation (No. 2015M582064). The author is a doctoral researcher in center for post-doctoral studies of Qufu Normal University. 


\section{Competing Interests}

The authors declare that they have no conflict of interests.

\section{References}

[1] Schmidli, H. (2002) On Minimizing the Ruin Probability by Investment and Reinsurance. Annals of Applied Probability, 12, 890-907.

https://doi.org/10.1214/aoap/1031863173

[2] Bai, L. and Guo, J. (2008) Optimal Proportional Reinsurance and Investment with Multiple Risky Assets and No-Shorting Constraint. Insurance: Mathematics and Economics, 42, 968-975. https://doi.org/10.1016/j.insmatheco.2007.11.002

[3] Chen, S., Li, Z. and Li, K. (2010) Optimal Investment-Reinsurance Policy for an Insurance Company with VaR Constraint. Insurance: Mathematics and Economics, 47, 13-24. https://doi.org/10.1016/j.insmatheco.2010.06.002

[4] Bäuerle, N. (2005) Benchmark and Mean-Variance Problems for Insurers. Mathematical Methods of Operations Research, 62, 159-165. https://doi.org/10.1007/s00186-005-0446-1

[5] Bai, L. and Zhang, H. (2008) Dynamic Mean-Variance Problem with Constrained Risk Control for the Insurers. Mathematical Methods of Operations Research, 68, 181-205. https://doi.org/10.1007/s00186-007-0195-4

[6] Zeng, Y. and Li, Z. (2011) Optimal Time-Consistent Investment and Reinsurance Policies for Mean-Variance Insurers. Insurance: Mathematics and Economics, 49, 145-154. https://doi.org/10.1016/j.insmatheco.2011.01.001

[7] Yang, H. and Zhang, L. (2005) Optimal Investment for Insurer with Jump-Diffusion Risk Process. Insurance: Mathematics and Economics, 37, 615-634.

https://doi.org/10.1016/j.insmatheco.2005.06.009

[8] Wang, N. (2007) Optimal Investment for an Insurer with Exponential Utility Preference. Insurance: Mathematics and Economics, 20, 77-84. https://doi.org/10.1016/j.insmatheco.2006.02.008

[9] Xu, L., Wang, R. and Yao, D. (2008) On Maximizing the Expected Terminal Utility by Investment and Reinsurance. Journal of Industrial and Management Optimization, 4, 801-815. https://doi.org/10.3934/jimo.2008.4.801

[10] Maenhout, P.J. (2004) Robust Portfolio Rules and Asset Pricing. Review of Financial Studies, 17, 951-983. https://doi.org/10.1093/rfs/hhh003

[11] Liu, H. (2010) Robust Consumption and Portfolio Choice for Time Varying Investment Opportunities. Annals of Finance, 6, 435-454. https://doi.org/10.1007/s10436-010-0164-4

[12] Yi, B., Li, Z., Viens, F.G. and Zeng, Y. (2013) Robust Optimal Control for an Insurer with Reinsurance and Investment under Hestons Stochastic Volatility Model. Insurance: Mathematics and Economics, 53, 601-614.

https://doi.org/10.1016/j.insmatheco.2013.08.011

[13] Li, Z., Zeng, Y. and Lai, Y. (2013) Optimal Time-Consistent Investment and Reinsurance Strategies for Insurers under Hestons SV Model. Insurance: Mathematics and Economics, 51, 191-203. https://doi.org/10.1016/j.insmatheco.2011.09.002

[14] Sun, Z., Zheng, X. and Zhang, X. (2017) Robust Optimal Investment and Reinsurance of an Insurer under Variance Premium Principle and Default Risk. Journal of Mathematical Analysis Applications, 446, 1666-1686.

https://doi.org/10.1016/j.jmaa.2016.09.053 
[15] Yi, B., Viens, F.G., Law, B. and Li, Z. (2015) Dynamic Portfolio Selection with Mispricing and Model Ambiguity. Annals of Finance, 11, 37-75. https://doi.org/10.1007/s10436-014-0252-y

[16] Gu, A., Viens, F.G. and Yi, B. (2017) Optimal Reinsurance and Investment Strategies for Insurers with Mispricing and Model Ambiguity. Insurance: Mathematics and Economics, 72, 235-249. https://doi.org/10.1016/j.insmatheco.2016.11.007

[17] Zeng, Y., Li, D., Chen, Z. and Yang, Z. (2018) Ambiguity Aversion and Optimal Derivative-Based Pension Investment with Stochastic Income and Volatility. Journal of Economic Dynamics and Control, 88, 70-103.

[18] Gu, A., Viens, F.G. and Yao, H. (2018) Optimal Robust Reinsurance-Investment Strategies for Insurers with Mean Reversion and Mispricing. Insurance: Mathematics and Economics, 80, 93-109. https://doi.org/10.1016/j.insmatheco.2018.03.004

[19] Ksendal, B. (1995) Stochastic Differential Equations: An Introduction with Applications. Springer-Verlag, Berlin.

[20] Zeng, Y. (2015) Robust Optimal Strategies for an Insurer with Reinsurance and Investment under Benchmark and Mean-Variance Criteria. Scandinavian Actuarial Journal, 8, 1-27.

[21] Anderson, E.W., Hansen, L.P. and Sargent, T.J. (2000) Robustness, Detection and the Price of Risk. 